Revista

Multi-Ensayos

Vol. 5, $\mathrm{N}^{\circ} 10$

ISSN: 2412-3285

https://multiensayos.unan.edu.ni

DOI: https://doi.org/10.5377/multiensayos.v5i10.8876

\title{
Estudio del desarrollo de competencias a través de prácticas experimentales. Experiencia en la asignatura de introducción a la química
}

\section{Study of skills development through experimental practices. Experience in the subject of introduction to chemistry}

Yadith Herrera Corrales ${ }^{1}$

Recibido: 24 de mayo de 2019. Aceptado: 10 de junio de 2019

\section{RESUMEN}

Las metas de todo sistema educativo es la formación de individuos preparados para enfrentar las diversas demandas de las empresas y sociedad en que se desenvuelven. Por ello los egresados de estudios superiores deben poseer capacidades críticas, analíticas, capaces de resolver problemas y la toma de decisiones para coadyuvar al desarrollo económico y social. Un profesional es competente, cuando adquiere los conocimientos tanto académicos, como habilidades y capacidades, aplicándolos con eficacia durante el desarrollo de actividades profesionales y su vida cotidiana. Es importante mencionar que las competencias genéricas o básicas, son en su mayoría imprescindible para poder alcanzar las competencias específicas. Por ello se debe tener presente que es ineludible el desarrollo de competencias básicas y no es posible omitir su fortalecimiento al introducir el aprendizaje de competencias específicas.

Palabras claves: competencias; desarrollo profesional.

\section{ABSTRACT}

The goals of every educational system is the formation of individuals prepared to face the various demands of the companies and society in which they operate. For this reason, graduates of higher education must have critical, analytical, problem-solving skills and decision-making to help economic and social development. A professional is competent, when he acquires the academic knowledge, skills and abilities, applying them effectively during the development of professional activities and their daily life. It is important to mention that generic or basic competences are mostly essential to achieve specific competences. It should therefore be borne in mind that the development of core competences is inescapable and its strengthening cannot be omitted by introducing skills-specific learning.

Keywords: competencies; professional development.

1 Docente UNAN-Managua/FAREM-Estelí. Correo electrónico: yadithherrera@yahoo.es. (c) 2019 Revista Multi-Ensayos. 


\section{INTRODUCCIÓN}

"Ser competente en un ámbito determinado es tener conocimientos necesarios para desenvolverse en ese ámbito y ser capaz de utilizarlos actuando con eficacia en el desarrollo de actividades y tareas relacionadas con él". (C. Coll y A. Marchesi, 2007, citado por Álvarez, S., Pérez, A. y Suárez M. 2008. p. 19)

"Competencia es la habilidad para afrontar demandas externas o desarrollar actividades y proyectos de manera satisfactoria en contextos complejos, implica dimensiones cognitivas y no cognitivas: conocimientos, habilidades cognitivas, habilidades prácticas, actitudes, valores y emociones" (A.I. Pérez Gómez, 2007, citado por Álvarez, S., Pérez, A. y Suárez M. 2008. p. 19)

Es relevante hacer notar que este segundo concepto no limita las competencias al área cognitiva, como lo hace el primer concepto. Los autores hacen referencia de las competencias desde la dimensión cognitiva y no cognitiva, incluyendo actitudes, valores y emociones, siendo estos tres últimos factores muy relevantes en el éxito profesional y en la vida cotidiana, ya que individuos faltos de valores éticos y morales, con actitudes y emociones negativas, difícilmente podrán obtener resultados eficaces y eficientes en el contexto donde se desarrollen.

\section{DESARROLLO}

García, J. y Mendoza, V. (2012) establecen que las competencias se clasifican de acuerdo al campo de aplicación en:

- Competencias genéricas o básicas, se refieren a las competencias que se aplican en todos los campos formativos y profesionales.

- Competencias específicas, son aquellas propias de una carrera o disciplina.

Considerando lo expuesto anteriormente se decide indagar sobre la incidencia que proveen las prácticas de laboratorio en la asignatura de Introducción a la Química en el desarrollo de conocimientos científicos, y habilidades técnicas en el uso de instrumentos y procedimientos experimentales. Esta experiencia se realiza en la UNAN -Managua, FAREM- Estelí, con un grupo de estudiantes de I Año de Ingeniería Agroindustrial. La información es recopilada del desarrollo de prácticas experimentales en la asignatura de Introducción a la Química.

Se elige a este grupo de estudiantes por su perfil profesional, que se enfoca principalmente en los procesos industriales de materia prima de origen agropecuario. Se entiende que los estudiantes deben desarrollar competencias específicas en el campo agroindustrial, sin embargo es fundamental que los estudiantes posean competencias específicas relacionadas a la asignatura de Introducción a la Química, la cual es la base de las transformaciones en los procesos productivos agroindustriales. 
La información es obtenida a partir de tres instrumentos que se aplicaron a los estudiantes después del desarrollo de primera práctica de laboratorio y posterior a la última práctica de laboratorio. A continuación se describen y analizan los resultados obtenidos.

Se desarrolla la primera práctica de laboratorio "Identificación de instrumentos de laboratorio y técnicas de mediciones de volumen y masa", el día 15/01/19, que es dirigida al curso de I Año de Ingeniería Agronómica.

Los objetivos que se espera alcancen los estudiantes son los siguientes:

- Identificar los instrumentos de laboratorio para realizar mediciones de volumen usando técnicas adecuadas.

- Aprender técnicas básicas para mediciones de volumen y masa.

- Adquirir destreza en el uso y manejo de instrumentos de laboratorio.

Las destrezas que se pretenden desarrollar en los estudiantes a través de las prácticas experimentales son:

- Nombrar correctamente los instrumentos de medición volumétrica.

- Identificar los instrumentos volumétricos con mediciones exactas.

- Realizar lecturas de volumen de forma correcta.

- Realizar determinaciones de densidad de líquidos y sólidos.

Este conjunto de destrezas brindarán a los estudiantes una base complementaria para el desarrollo de competencias específicas en el área de la Química, que es importante en el perfil profesional de los Ingenieros Agroindustriales.

Posterior a la práctica experimental se aplicaron dos instrumentos a un total de 30 estudiantes, cada instrumento fue completado por 15 estudiantes seleccionados de forma aleatoria. El instrumento 1 indaga la percepción que tienen los estudiantes sobre la asignatura de Introducción a la química y otros 15 estudiantes llenaron el instrumento 2, el cual evalúa los aprendizajes técnicos adquiridos durante la práctica de laboratorio No.1.

Resultados obtenidos del instrumento 1 "Percepción de los e estudiantes con respecto a la asignatura de Introducción a la Química"

\begin{tabular}{lr}
\hline Considera difícil la asignatura de Química & Cantidad \\
\hline Si & 7 \\
No opino / no sé & 4 \\
Depende del estudiante & 3 \\
No & 1 \\
\hline Tabla 1. Instrumento 1. Dificultad de la asignatura de Química.
\end{tabular}




\begin{tabular}{lr}
\hline \multicolumn{1}{c}{ ¿Qué es lo más difícil? } & Cantidad \\
\hline Ejercicios y formulas & 6 \\
Teoría & 3 \\
No opino / no sé & 3 \\
Todo & 2 \\
Experimentos & 1 \\
\hline
\end{tabular}

Tabla 2. Instrumento 1. Lo más difícil en Química.

\begin{tabular}{|c|c|}
\hline Lo que gusta más & Cantidad \\
\hline Prácticas de laboratorio & 10 \\
\hline Todo & 1 \\
\hline Contenido & 1 \\
\hline Metodología & 1 \\
\hline Nada & 1 \\
\hline No opino & 1 \\
\hline
\end{tabular}

Tabla 3. Instrumento 1. Lo que gusta más de Química.

\begin{tabular}{lr}
\hline \multicolumn{1}{c}{ Sugerencias } & Cantidad \\
\hline Realizar más prácticas de laboratorio & 5 \\
Ninguna, todo está bien & 5 \\
Explicar más despacio y repasar & 2 \\
Dinamismo & 1 \\
Poner atención & 1 \\
No opino & 1 \\
\hline
\end{tabular}

Tabla 4. Instrumento 1. Sugerencias.

\begin{tabular}{lr}
\hline \multicolumn{1}{c}{ ¿Qué estás dispuesto hacer? } & Cantidad \\
\hline Echarle ganas & 6 \\
Poner atención & 5 \\
Estudiar & 4 \\
Cumplir con la tarea & 2 \\
Practicar los ejercicios & 2 \\
Buscar información & 1 \\
Puntualidad & 1 \\
\hline
\end{tabular}

Tabla 5. Instrumento 1. Disponibilidad de los estudiantes.

*Las cantidades son el número de menciones de cada acción

\section{Análisis de resultados del instrumento 1 "Percepción de los estudiantes con respecto a la asignatura de Introducción a la Química"}

Al menos la mitad de los estudiantes consideran la asignatura de Introducción a la Química compleja y con un grado alto de dificultad, coincidiendo que lo más difícil son los ejercicios y la aplicación de fórmulas. Con respecto a las estrategias aplicadas, la tercera parte de los estudiantes expresan sentirse 
satisfechos, en cambio la otra tercera parte de estudiantes mencionan que es necesario explicar más despacio, repasar y prestar atención. Lo que hace necesario una revisión de la transposición didáctica que se está realizando con los contenidos de la asignatura y principalmente adecuar las estrategias aplicadas en el aula, dándoles un rol mucho más activo a los estudiantes.

En cuanto a la realización de prácticas de laboratorio aproximadamente un $70 \%$ refieren que es lo que más le gusta de esta asignatura. Y la tercera parte de los estudiantes mencionan que se deben realizar más prácticas de laboratorio como sugerencia. Posteriormente se analizan los resultados del aprendizaje alcanzado en la primera práctica de laboratorio.

Es interesante la disponibilidad de los estudiantes donde se observa que un poco más de la mitad de los estudiantes están dispuestos a trabajar duro para aprender, poniendo atención y estudiando, sin embargo son pocos los que mencionan el cumplimiento de tareas y practica en casa los ejercicios, y aún menos estudiantes reconocen la búsqueda de información como una actividad importante. Esto permite visualizar que es necesario promover la búsqueda de información, resolución de ejercicios de tal manera que resulten motivadores para los estudiantes, considerando en la planeación y el diseño de actividades de aprendizaje, la motivación por logro alcanzado, lo que por ende incentivará el cumplimiento de la tareas que es una herramienta muy importante en el aprendizaje.

Resultados obtenidos del instrumento 2 "Evaluación de aprendizajes alcanzados en la práctica de laboratorio No. 1"

\begin{tabular}{lrr}
\hline Identificar instrumentos & Cantidad & Porcentaje \\
\hline Identifica 0 instrumentos de 6 & 1 & $7 \%$ \\
Identifica 1 instrumentos de 6 & 1 & $7 \%$ \\
Identifica 2 instrumentos de 6 & 0 & $0 \%$ \\
Identifica 3 instrumentos de 6 & 4 & $27 \%$ \\
Identifica 4 instrumentos de 6 & 6 & $40 \%$ \\
Identifica 5 instrumentos de 6 & 2 & $13 \%$ \\
Identifica 6 instrumentos de 6 & 1 & $7 \%$ \\
\hline
\end{tabular}

Tabla 6. Instrumento 2. Identificar instrumentos volumétricos.

\begin{tabular}{lrr}
\hline $\begin{array}{c}\text { Conocer instrumentos con medidas de } \\
\text { volumen exactas }\end{array}$ & Cantidad & Porcentaje \\
\hline Identifica 0 instrumentos de 4 & 13 & $87 \%$ \\
Identifica 1 instrumentos de 4 & 0 & $0 \%$ \\
Identifica 2 instrumentos de 4 & 2 & $13 \%$ \\
Identifica 3 instrumentos de 4 & 0 & $0 \%$ \\
\hline
\end{tabular}

Tabla 7. Instrumento 2. Identificar instrumentos volumétricos exactos. 


\begin{tabular}{lr}
\hline \multicolumn{1}{c}{ Explicar técnica de lectura de volumen } & Cantidad \\
\hline Explicación correcta & 4 \\
Explicación incorrecta o no responde & 11 \\
\hline
\end{tabular}

Tabla 8. Instrumento 2. Técnica de lectura de volumen.

\begin{tabular}{lrr}
\hline \multicolumn{1}{c}{ Realizar lectura de volumen } & Cantidad & Porcentaje \\
\hline Lectura de volumen correcta & 0 & $0 \%$ \\
Lectura de volumen incorrecta o no responde & 15 & $100 \%$ \\
\hline
\end{tabular}

Tabla 9. Instrumento 2. Lectura de volumen.

\begin{tabular}{lr}
\hline \multicolumn{1}{c}{ Explicar determinación de densidad } & Cantidad \\
\hline Explicación corrrecta & 0 \\
Explicación incorrecta o no & 15 \\
\hline
\end{tabular}

Tabla 10. Instrumento 2. Determinación de densidad.

Análisis de resultados del instrumento 2 "Evaluación de aprendizajes alcanzados en la práctica de laboratorio No. 1"

Partiendo de los objetivos que se plantearon en esta práctica de laboratorio que son:

- Identificar los instrumentos de laboratorio para realizar mediciones de volumen usando técnicas adecuadas.

- Aprender técnicas básicas para mediciones de volumen y masa.

- Adquirir destreza en el uso y manejo de instrumentos de laboratorio.

Y las siguientes destrezas que se pretenden desarrollar:

- Nombrar correctamente los instrumentos de medición volumétrica.

- Identificar los instrumentos volumétricos con mediciones exactas.

- Realizar lecturas de volumen de forma correcta.

- Realizar determinaciones de densidad de líquidos y sólidos.

Se determinó en la tabla No. 6 que el 20\% de los estudiantes logran identificar entre 5 y 6 instrumentos de 6, que fueron utilizados en la práctica de laboratorio. En cuanto a identificar instrumentos de medición de volúmenes exactos se refleja poco conocimiento de ellos, por lo tanto es imprescindible desarrollar una actividad o tarea que permita el reconocimiento de estos instrumentos.

Sobre la técnica de lectura de volúmenes es notoria la poca apropiación de estos conocimientos técnicos, igual ocurre con la determinación práctica de la densidad de sustancias y cuerpos sólidos. Esto debe generar una alerta en el diseño y desarrollo de las prácticas de laboratorio, ya que el estudiante no logra apropiarse de los conocimientos técnicos y teóricos necesarios para alcanzar los objetivos y destrezas esperadas. 
El diseño de esta práctica de laboratorio es del tipo instruccional y contiene su fundamento teórico que lo sustenta, es decir se indica paso a paso el procedimiento a realizar, sin embargo a pesar de brindarse la información necesaria a los estudiantes se pudo notar que se presentaron dificultades para seguir las instrucciones, debido a que los estudiantes no interpretaban correctamente las indicaciones. Además se observa que los estudiantes no realizaron la lectura y posterior comprensión de la teoría que incluye la guía de laboratorio.

\section{Resultados obtenidos del instrumento No. 3 para evaluar el aprendizaje técnico necesario para el fortalecer competencias profesionales}

Durante el desarrollo de la asignatura se realizaron 3 prácticas de laboratorio, dos de ellas relacionadas con la identificación de instrumentos, determinación de volumen y masa, y determinación de densidad de líquidos y cuerpos sólidos. Y la tercera práctica experimental que se aplicó abordó el tema de reacciones químicas.

A pesar de tener claro la necesidad de realizar un número mayor de prácticas de laboratorio, el tiempo disponible para las mismas, fue poco debido a las dificultades que expresan los estudiantes sobre la comprensión de conceptos y teorías, además la resolución de ejercicios se les dificultó. Por ello se destinó más tiempo en recapitular los diferentes contenidos de la asignatura.

Este instrumento 3 se aplicó durante las últimas semanas de encuentros presenciales a 32 estudiantes que representan el $100 \%$ de estudiantes activos en el curso de I Año de Ingeniería Agroindustrial. Obteniéndose los siguientes resultados.

\begin{tabular}{lrr}
\hline Identificar instrumentos & Cantidad & Porcentaje \\
\hline Identifica 0 instrumentos de 6 & 0 & $0 \%$ \\
Identifica 1 instrumentos de 6 & 1 & $3 \%$ \\
Identifica 2 instrumentos de 6 & 1 & $3 \%$ \\
Identifica 3 instrumentos de 6 & 3 & $9 \%$ \\
Identifica 4 instrumentos de 6 & 6 & $19 \%$ \\
Identifica 5 instrumentos de 6 & 8 & $25 \%$ \\
Identifica 6 instrumentos de 6 & 13 & $41 \%$ \\
\hline
\end{tabular}

Tabla 11. Instrumento 3. Identificar instrumentos volumétricos.

\begin{tabular}{lrr}
\hline $\begin{array}{c}\text { Conocer instrumentos con medidas de } \\
\text { volumen exactas }\end{array}$ & Cantidad & Porcentaje \\
\hline Identifica 0 instrumentos de 4 & 25 & $78 \%$ \\
Identifica 1 instrumentos de 4 & 3 & $9 \%$ \\
Identifica 2 instrumentos de 4 & 2 & $6 \%$ \\
Identifica 3 instrumentos de 4 & 2 & $6 \%$ \\
\hline
\end{tabular}

Tabla 12. Instrumento 3. Identificar instrumentos volumétricos exactos. 


\begin{tabular}{lrr}
\hline \multicolumn{1}{c}{$\begin{array}{c}\text { Identificar unidades de medidas de los } \\
\text { instrumentos de volumen utilizados }\end{array}$} & Cantidad & Porcentaje \\
\hline Expresa de forma correcta & 16 & $52 \%$ \\
Expresa el tipo de unidad de medida (volumen) & 6 & $19 \%$ \\
No responde o lo hace de forma incorrecta & 9 & $29 \%$ \\
\hline
\end{tabular}

Tabla 13. Instrumento 3. Identificar unidad de medida de instrumento volumétrico.

\begin{tabular}{lrr}
\hline \multicolumn{1}{c}{$\begin{array}{c}\text { Identificar unidad de medida de la balanza } \\
\text { utilizada }\end{array}$} & Cantidad & Porcentaje \\
\hline Expresa de forma correcta & 19 & $59 \%$ \\
Expresa la unidad de medida de kg & 5 & $16 \%$ \\
Expresa el tipo de unidad de medida (masa) & 1 & $3 \%$ \\
No responde o lo hace de forma incorrecta & 7 & $22 \%$ \\
\hline
\end{tabular}

Tabla 14. Instrumento 3. Identificar unidad de medida de balanza.

\begin{tabular}{lrr}
\hline \multicolumn{1}{c}{ Realizar lectura de volumen } & Cantidad & Porcentaje \\
\hline Lectura de volumen correcta & 11 & $34 \%$ \\
Lectura de volumen incorrecta o no responde & 21 & $66 \%$ \\
\hline
\end{tabular}

Tabla 15. Instrumento 3. Lectura de volumen.

\begin{tabular}{lrr}
\hline \multicolumn{1}{c}{ Identificar reacciones químicas } & Cantidad & Porcentaje \\
\hline Expresa que ocurren cambios sin especificarlos & 5 & $16 \%$ \\
Expresa como cambio único el cambio de color & 4 & $13 \%$ \\
Expresa como cambios, el color y textura & 1 & $3 \%$ \\
No responde o su respuesta es incorrecta & 22 & $69 \%$ \\
\hline
\end{tabular}

Tabla 16. Instrumento 3. Identificar reacciones químicas.

\begin{tabular}{lrr}
\hline \multicolumn{1}{c}{ Preparación de disoluciones } & Cantidad & Porcentaje \\
\hline $\begin{array}{l}\text { Describe el proceso sin especificar técnicas } \\
\text { necesarias }\end{array}$ & 2 & $6 \%$ \\
No responde o su respuesta es incorrecta & 30 & $94 \%$ \\
\hline
\end{tabular}

Tabla 17. Instrumento 3. Preparación de disoluciones.

\section{Análisis de resultados del instrumento No. 3 para evaluar el aprendizaje técnico necesario para el fortalecer competencias profesionales}

Al comparar los resultados de los instrumentos 2 y 3 sobre los aprendizajes y destrezas alcanzadas con respecto a las siguientes destrezas:

- Nombrar correctamente los instrumentos de medición volumétrica.

- Identificar los instrumentos volumétricos con mediciones exactas.

- Realizar lecturas de volumen de forma correcta.

- Realizar determinaciones de densidad de líquidos y sólidos. 
La identificación de los diferentes instrumentos volumétricos, se determinó que un 66 \% de estudiantes lograron identificar entre 5 y 6 instrumentos de los 6 instrumentos de medición volumétrica al finalizar la asignatura de Introducción a la Química. En cambio el porcentaje inicial fue de un $20 \%$ siendo el resultado de la primera práctica de laboratorio (Instrumento 2). Es decir que un $46 \%$ de estudiantes adquirieron destreza en la identificación de los instrumentos volumétricos.

Sin embargo los estudiantes que no alcanzaron los aprendizajes y destrezas esperados, son consecuencia de una serie de factores, entre ellos se menciona el de mayor relevancia y el más determinante:

Los estudiantes poseen debilidades en competencias genéricas o básicas indispensables para el desarrollo de habilidades y destrezas necesarias en la formación de competencias específicas. La principal competencia que afecta el aprendizaje de los estudiantes es "el pensamiento crítico", de acuerdo con Paul y Elder (2005), citado por García, J. y Mendoza, V. (2012), el pensamiento crítico es importante en el proceso educativo ya que:

- Lleva al dominio del contenido y al aprendizaje profundo.

- Desarrollo la apreciación por la razón y la evidencia.

- Anima a los estudiantes a descubrir y a procesar la información.

- Enseña a los estudiantes a pensar y a formular conclusiones.

- Muestra cómo defender posiciones en asuntos complejos, considerar una amplia variedad de puntos de vista, y analizar conceptos, teorías y explicaciones.

- Enseña a los estudiantes a aclarar asuntos y conclusiones, a resolver problemas, a transferir ideas a nuevos contextos y a examinar suposiciones, a evaluar hechos supuestos, a explorar implicaciones. (p.33)

Facione, Peter A. (2007) establece seis habilidades cognitivas que son esenciales en el pensamiento crítico. A continuación se describen cada una de ellas.

1. La interpretación: Consiste en "comprender y expresar el significado o la relevancia" de la información.

2. Análisis: Es identificar las relaciones de inferencias reales y supuestas entre enunciados, preguntas, conceptos, descripciones u otras formas de representación que tienen el propósito de expresar creencia, juicio, experiencias, razones, información u opiniones.

3. Evaluación: Se refiere a valorar inferencia realizada anteriormente en el análisis con respecto a la relación real o supuesta entre los enunciados, preguntas, creencias, entre otros.

4. Inferencia: Es la capacidad de establecer el vínculo de lo presentado con los resultados probables. Aquí tenemos la formulación de hipótesis y el obtener conclusiones en forma anticipada.

5. Explicación: La persona que ha razonado y entendido una situación, ha comprendido las causas y los efectos. Puede describir adecuadamente los pasos que se siguen en un determinado procedimiento, es capaz de comunicar por escrito o verbalmente en forma clara un juicio reflexivo de algún tema. Lo entiende y se da a entender, esa persona habrá dominado la habilidad de la explicación.

6. Autorregulación. Esta habilidad es de orden superior, se le pueda llamar Meta cognición. Su aplicación 
consiste en que el pensador evalúa sus propios pensamientos, juzga su trabajo y lo puede corregir si ve que hay la necesidad de hacerlo. Modifica, corrige o amplía sus enunciados, los revisa y los coteja. No espera que le digan que hacer, lo hace por cuenta propia. Estudia, investiga, se informa, dispone de sus horas de sueño y de comida. Escucha opiniones aun cuando no esté de acuerdo con ellas, sabe que le van a servir para ampliar sus conocimientos. La necesidad de saber y superarse es el motor.

De lo expuesto anteriormente, se visibiliza la importancia de la competencia básica "pensamiento crítico" en el proceso de aprendizaje dentro del sistema educativo que se enfoca en el desarrollo de competencias. Ya que el conjunto de habilidades incluidas en esta competencia son las que permiten que el estudiante logre aprendizajes significativos, y pueda fortalecer otras competencias básicas, y adquirir competencias específicas necesarias en la formación de futuros profesionales.

\section{CONCLUSIÓN}

Las prácticas de experimentales, son herramientas necesarias e ineludibles en el proceso de aprendizaje, principalmente cuando se establece el desarrollo de competencias. Sin embargo la aplicación de prácticas de laboratorio donde los estudiantes sigan instrucciones, no favorece el aprendizaje y el desarrollo de competencias. Esto obliga al docente a realizar una reestructuración en los diseños didácticos de estas actividades, considerando las deficiencias que pudieran tener los estudiantes en cuanto a las habilidades necesarias de la competencia básica "pensamiento crítico".

Es conveniente diseñar actividades experimentales que incluyan acciones previas, donde se definirá con claridad la intencionalidad de la experiencia, la vinculación de la teoría científica con la práctica experimental, en la cual los estudiantes podrán definir una hipótesis. Posteriormente el desarrollo acompañado de tareas que refuercen el aprendizaje que se desea alcanzar y finalmente incluir una actividad que permita el debate, análisis y reflexión sobre las conclusiones que generaran los estudiantes al finalizar la práctica de laboratorio.

El diseño de las guías de laboratorio requiere de una estructura que incluya tareas y actividades, que permitan el fortalecimiento de las habilidades y destrezas básicas, abordándolas de manera transversal. La identificación de las habilidades poco desarrolladas de la competencia "pensamiento crítico": interpretación, análisis, evaluación inferencia, explicación y autorregulación, es indispensable en la planeación de actividades experimentales. Es conveniente realizar una selección de estas habilidades y destrezas que sean inherentes al aprendizaje del contenido científico. Por ello la selección de estas tareas y actividades debe realizarse a partir del listado de habilidades y destrezas tanto básicas, como específicas. Este listado debe contener un número reducido de habilidades y destrezas, para que la complejidad del diseño de la práctica de laboratorio no sea excesiva. 


\section{REFERENCIAS}

Álvarez Morán, Sara, Pérez Collera, Arturo y Suarez Álvarez, María Luisa. (2008). Hacia un enfoque de la educación en competencias. Consejería de Educación y Ciencia del Principado de Asturias. España

Facione, Peter A. (2007). Pensamiento Crítico: ¿Qué es y por qué es importante?. Insigth Assessment. California Academic Press. Recuperado el 10 de Mayo 2019 de: http://www.eduteka.org/ PensamientoCriticoFacione.php

García de De León, Jaqueline y Mendoza Alvarado, Vivian. (2012). Desarrollo de Capacidades Metacognitivas y del Pensamiento Crítico en la Educación Básica ed. Vol. 53. Colección Pedagógica Formación Inicial de Docentes Centroamericanos de Educación Primaria o Básica. Coordinación Educativa y Cultural Centroamericana. San José, Costa Rica 